\title{
Editorial
}

\section{Multi-component Lifestyle Interventions for Diabetes and Associated Non-communicable Diseases: Considerations for Future Research}

\author{
Ahmad Alkhatib, PhD, MSc, RNutr, FRSM* \\ Professor of Health Nutrition and Sport Science, Institute of Sport Science, University of Taipei, Taipei, Taiwan, Republic of China
}

\section{"Corresponding author}

Ahmad Alkhatib, PhD, MSc, RNutr, FRSM

Professor of Health Nutrition and Exercise Science, Institute of Sport Science, University of Taipei, Shilin District, I I , Taipei, Taiwan, Republic of China; Tel. +886 (0) 934089846; E-mail: drahmadalkhatib@gmail.com

\section{Article information}

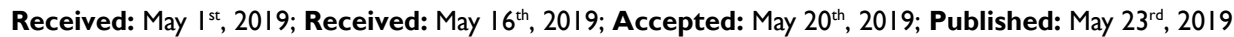

\section{Cite this article}

Alkhatib A. Multi-component lifestyle interventions for diabetes and associated non-communicable diseases: Considerations for future research. Obes Res Open J. 2019; 6(I): e5-e6. doi: I0.17|40/OROJ-6-e0I5

$\mathrm{D}$ abetes prevalence is at an alarming level. It is predicted that one in ten adults will have the condition by 2030 compared with one in eleven at present. ${ }^{1}$ Despite several global calls for action to reverse such a trend, the number of people with diabetes and associated lifestyle diseases continues to rise leading to significant health burdens, morbidity and premature mortality. ${ }^{2}$ Such challenge makes it crucial to adopt immediate preventative policies which embed effective lifestyle diabetes interventions, especially those integrating multi-components interventions. ${ }^{3}$

Adopting a multi-component approach which encompasses changing behavioral and physical aspects is likely to be more effective than a single component diabetes prevention program. Lessons from large lifestyle interventions combining a variety of physical activity patterns with different healthy dietary regimes have unequivocal evidence about their joint long-term effectiveness compared with adopting a single component whether exercise, diet or medication alone. ${ }^{4,5}$ For example, the Finish diabetes study was first to show a remarkable $58 \%$ reduced rates of diabetes incidence when a longitudinal multi-component intervention (behavioral, exercise and dietary) was followed by individuals with glucose intolerance. ${ }^{3}$ Similar longitudinal diabetes risk reduction of $58 \%$ was found for those with pre-diabetes in the US diabetes prevention program when exercise and diet approaches were combined, compared with almost half (30\% reduction) found when relying on insulin-sensitizing drug Metformin. ${ }^{5}$ A fifteen-year follow-up of the latter showed that medication did not elicit better risk-reduction benefits than a multi-component lifestyle intervention.

Adopting healthy behaviors, particularly adopting physi- cal activity and healthy nutrition can have reciprocal favorable outcomes. Observational evidence has reported that amongst older adults (60-80-years-old), those who consumed a healthy diet such as the Mediterranean diet were more likely to be active in their lifestyle than those who consumed a western diet. ${ }^{6}$ In such, adopting such a dietary component with an exercise training component provided additional vascular and cardiometabolic protective benefits in high-risk individuals. ${ }^{7,8}$ On the other hand, adding an exercise to a multi-nutrient supplementation proved much more effective in preventing age-related decline in high-risk very old frail men and women, than the supplement alone, a single intervention component. ${ }^{9}$ The latter study showed that a single component (supplementation only) failed to slow age-related deleterious outcomes compared with favorable outcomes found when strength exercise was combined with the supplementation. ${ }^{9}$ In such, interventions should always adopt exercise and nutrition as essential components of a lifestyle intervention strategy. Even at the screening phase, assessing a combined physical activity, cardiorespiratory fitness, nutritional intake alongside diabetes and associated cardiometabolic risk factors, is likely to encourage healthy behavior change in high-risk individuals. ${ }^{10,11}$ Therefore, adopting multi-component prevention strategy, both at baseline assessment and throughout the intervention not only augments their benefits but prevents potential hazards.

Addressing as many lifestyle components as feasible is also important. For example, it is now known that sedentary behavior and sleep patterns are associated with the development of diabetes. ${ }^{12}$ Recent research evidence has classified sedentary lifestyle behavior, such as TV watching, prolonged sitting hours or driving as an independent risk factor for type-II diabetes. ${ }^{12,13}$ Reported as- 
sociations include higher type-2 diabetes incidents, cardiovascular disease and all-cause mortality independently of leisure-time physical activity ${ }^{13}$ Since it is difficult to quantify such behaviors in physiological terms, cardiorespiratory fitness remains the gold standard for measuring physical activity or its lack, since that least cardiorespiratory fit individuals suffer from significantly increased chronic disease risks and all cause-mortality risks. ${ }^{14}$ Therefore, sedentary behaviors, physical activity, and cardiovascular health can all be assessed through cardiorespiratory fitness as an essential vital sign, ${ }^{15}$ and so it should form an integral part of any lifestyle intervention.

The scientific knowledge about the behavioral, lifestyle and biological components is continuously evolving, which enables better targeting of several modifiable disease risk factors for diabetes and associated diseases. This also requires integrating exercise science, nutrition and behavior approaches to personalize future lifestyle interventions. Future studies can take forward previous success stories using multi-component lifestyle interventions based on contemporary knowledge and recent technological advances relevant to the $21^{\text {st }}$ century.

\section{REFERENCES}

1. International Diabetes Federation. Diabetes Atlas $7^{\text {th }}$ Edition. 2015. Web site. http://www.diabetesatlas.org/. Accessed April 1, 2019.

2. World Health Organization (WHO). Global Report on Diabetes. 2016. Web site: http://www.who.int/diabetes/global-report/ en/. Accessed April 1, 2019.

3. Alkhatib A, Tuomilehto J. Lifestyle diabetes prevention. In: Huhtaniemi I, Martini L, eds. Encyclopaedia of Endocrine Diseases, $2^{\text {nd }}$ ed. Amsterdam, Netherlands: Elsevier; 2018.

4. Tuomilehto J, Lindstrom J, Eriksson JG, et al. Prevention of type 2 diabetes mellitus by changes in lifestyle among subjects with impaired glucose tolerance. N Engl J Med. 2001; 344(18): 13431350. doi: 10.1056/NEJM200105033441801

5. Knowler WC, Barrett-Connor E, Fowler SE, et al. Reduction in the incidence of type 2 diabetes with lifestyle intervention or metformin. N Engl J Med. 2002; 346(6): 393-403. doi: 10.1056/ NEJMoa012512

6. Bibiloni MDM, Julibert A, Argelich E, et al. Western and medi- terranean dietary patterns and physical activity and fitness among Spanish older Adults. Nutrients. 2017; 9(7): pii: E704. doi: 10.3390/ nu9070704

7. Alkhatib A. Effective intervention strategies combining Mediterranean diet and exercise for reducing obesity, metabolic and cardiovascular risks in high-risk populations: Mini review. Obes Res Open J. 2015; 1(1): 4-9. doi: 10.17140/OROJ-1-102

8. Alkhatib A, Klonizakis M. Effects of exercise training and Mediterranean diet on vascular risk reduction in post-menopausal women. Clin Hemorheol Microcirc. 2014; 57(1): 33-47. doi: 10.3233/ CH-131770

9. Fiatarone MA, O’Neill EF, Ryan ND, et al. Exercise training and nutritional supplementation for physical frailty in very elderly people. N Engl J Med. 1994;330(25):1769-75. doi: 10.1056/ NEJM199406233302501

10. Alkhatib A. Sedentary risk factors across genders and job roles within a university campus workplace: Preliminary study. J Occup Health. 2013; 55: 218-224. doi: 10.1539/joh.12-0158-OA

11. Alkhatib A. High prevalence of sedentary risk factors amongst university employees and potential health benefits of campus workplace exercise intervention. Work. 2015; 52(3): 589-595. doi: 10.3233/WOR-152182

12. Alkhatib A. Sedentary Lifestyle, Predictive Factors, Health Risks and Physiological Implications. New York, USA: Nova Science Publishers; 2016.

13. Biswas A, Oh PI, et al. Sedentary time and its association with risk for disease incidence, mortality, and hospitalization in adults: A systematic review and meta-analysis. Ann Intern Med. 2015; 162(2): 123-132. doi: 10.7326/M14-1651

14. Blair SN, Brodney S. Effects of physical inactivity and obesity on morbidity and mortality: Current evidence and research issues. Med Sci Sports Exerc. 1999; 31(Suppl): S646-S662. doi: 10.1097/00005768-199911001-00025

15. Despres JP. Physical activity, sedentary behaviours, and cardiovascular health: When will cardiorespiratory fitness become a vital sign? Can J Cardiol. 2016; 32(4): 505-513. doi: 10.1016/j. cjca.2015.12.006 